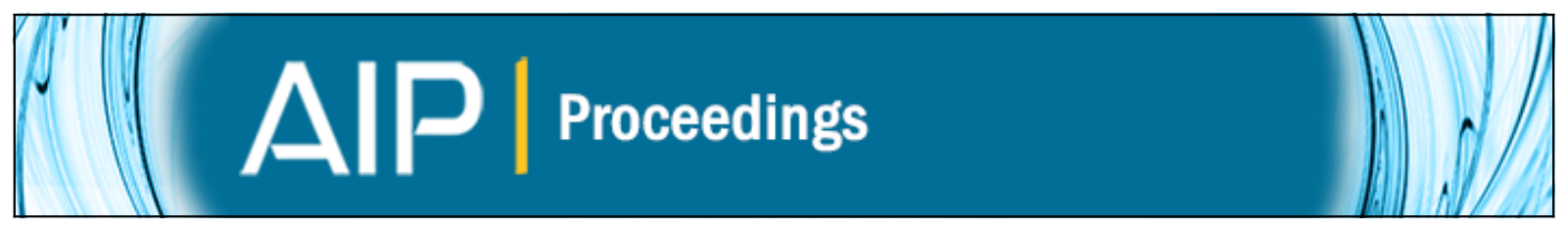

Doping Distribution Of An Operating Organic LightEmitting Diode: A Raman Map Analysis

BeynorAntonio PaezSierra, Henrique Leonel Gomes, and VictorTapio RangelKuoppa

Citation: AIP Conference Proceedings 1399, 875 (2011); doi: 10.1063/1.3666655

View online: http://dx.doi.org/10.1063/1.3666655

View Table of Contents: http://scitation.aip.org/content/aip/proceeding/aipcp/1399?ver=pdfcov

Published by the AIP Publishing 


\title{
Doping Distribution Of An Operating Organic Light- Emitting Diode: A Raman Map Analysis
}

\author{
Beynor-Antonio Paez-Sierra ${ }^{1}$, Henrique Leonel Gomes ${ }^{2}$ and Victor-Tapio Rangel- \\ Kuoppa $^{3}$ \\ ${ }^{I} R \& D$ Nanophotonics, QUBITON Laboratories, A-4040 Linz, Austria \\ ${ }^{2}$ Universidade do Algarve, Center of Electronic Optoelectronics and Telecommunications (CEOT), Campus de \\ Gambelas, 8000 Faro, Portugal \\ ${ }^{3}$ Institute of Semiconductor and Solid State Physics, Johannes Kepler Universität, A-4040 Linz, Austria
}

\begin{abstract}
We present confocal Raman spectroscopy (CSRS) maps of Poly(9,9-dioctylfluorene) (PFO)-based organic light emitting diode under operation. The CSRS analysis of the OLEDs was performed in normal room conditions. The non-emissive spots presented higher Raman intensity and broadening of the vibrational bands in comparison with the luminescent ones. The phenomenon is associated with an increase in the PFO $\pi-\pi^{*}$ absorption band and hence modification of the PFO doping which becomes favorable to the excitation wavelength, thus the Raman spectrum is enhanced. To the authors' knowledge this image technique had been missed for the OLED technology
\end{abstract}

Keywords: Organic electronics, Raman spectroscopy, PFO, OLEDs, organic semiconductors.

PACS: 78.30.-j, 78.66.Qn, 72.80.Le, 73.61.Ph, 77.55.dj.

\section{INTRODUCTION}

OLEDs have already matured for commercial applications. Some of the long-standing challenges in OLED technology relay on degradation and failure mechanisms. Thus the issue of lifetime presents great challenges. Confidence on device performance, interlayer identity during OLED operation is always desired. OLED performance and their degradation have been investigated by using a large number of techniques including micro Raman spectroscopy [1], surface-enhanced Raman spectroscopy (SERS) [2], Infrared spectroscopy [3], electronic-based and contact-based probes [4]. Particularly, implementation of non-invasive optical-based probes such as Raman spectroscopy for in situ investigation of charge states, crystallinity, local temperature conditions, or interface profiling of organic-based devices is of great interest $[1,5]$. Raman is a powerful tool based on the vibrational properties of matter. It gives better confidence on the molecular identity at different surfaces, interfaces, and operating conditions [2,4]. Moreover, it helps for an efficient detection of several effects, such as morphological changes, formation of new chemical species or chemical bonds between the metal contact and the active material [6].
The non-invasive vibrational analysis permits energetically to resolve fine-structure features, e.g. a $1000 \mathrm{~cm}^{-1}$ Raman shift corresponds to about 123.9 $\mathrm{meV}$. Here the analysis resolves energies as better as $10 \mathrm{meV}$.

\section{EXPERIMENTAL CONDITIONS}

The investigated OLEDs are glass/bottom electrode/organic layer/top electrode sandwich structures. The methods used for their fabrication are described in Ref. [7]. These devices consist of a $80 \mathrm{~nm}$ thick layer of green PFO acting as active layer and spincoated onto patterned $30 \mathrm{~nm}$ aluminum electrodes, previously deposited on the glass substrate. The device is completed by deposition of a transparent $30 \mathrm{~nm}$ barium electrode working as cathode, and then encapsulated. The active device is $3 \times 3 \mathrm{~mm}^{2}$.

The in situ analysis of the OLEDs was performed in normal room conditions. Samples were biased with a sub-femtoamp remote source meter model 6430 from Keithley. The CSRS investigations were carried out in a fully-atomized LabRAM ARAMIS spectrometer from Jobin Yvon HORIBA. The OLED active layer was analyzed in the back scattering configuration, and a $784.7 \mathrm{~nm}(1.58 \mathrm{eV})$ laser beam with an intensity of $0.1 \mathrm{~mW}$ was used as excitation source. The scheme of 
the OLED structure with the electrical connections and Raman optics is described in FIG. 1

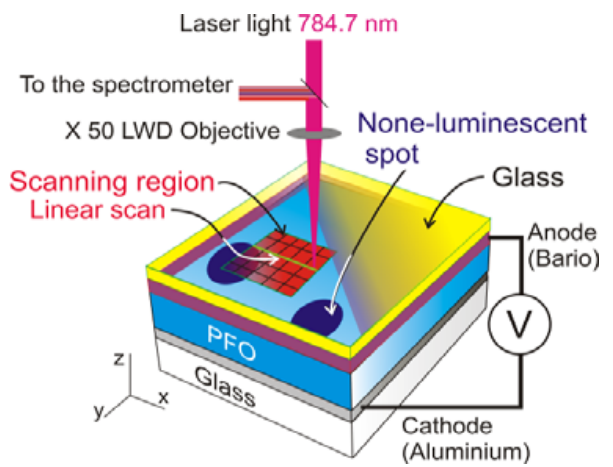

FIGURE 1. Experimental scheme of the OLED architecture and Raman optics in the back-scattering geometry.

\section{RESULTS AND CONCLUSIONS}

FIG. 2 presents a typical linear CSRS map performed from emissive points of the PFO layer towards non-emissive ones. The progressive band broadening from emissive spots towards non-emissive zones is well-pronounced in the spectral region [1300$1600 \mathrm{~cm}^{-1}$. The linear scan indicates that the transition from emissive to non-emissive spots occurs in a gradual manner accompanied with an increase of the Raman intensity. The latter phenomenon is associated with increase in the PFO $\pi-\pi^{*}$ absorption band and hence with modification of the PFO doping, which becomes favorable to the excitation wavelength (784.7 $\mathrm{nm})$. The Raman intensity of the none-emissive regions is higher in comparison with the emissive spots. Similar results based on surface-enhanced Raman spectroscopy (SERS) performed in ITO/P3OT/Al revealed SERS effect in combination with reduction of the emission power [2].

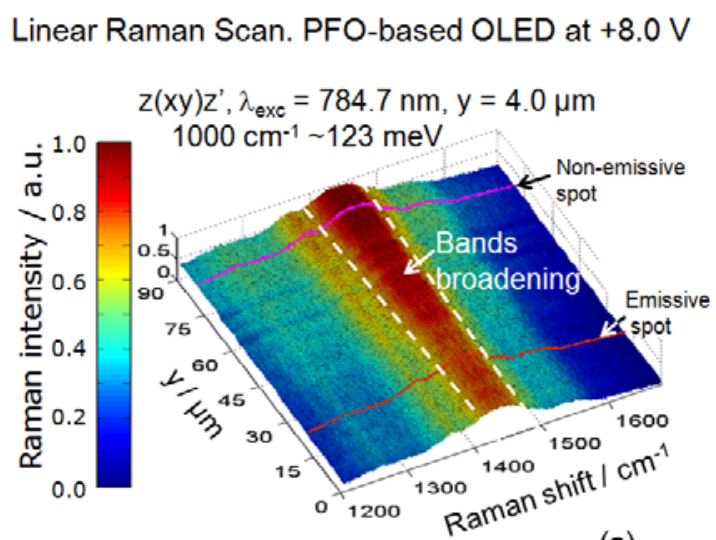

(a)

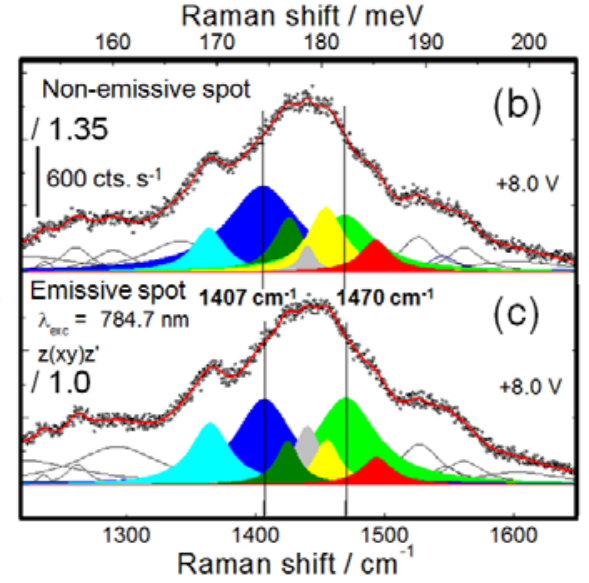

FIGURE 2. Linear CSRS of the PFO-based OLED operating at $+8.0 \mathrm{~V}$. (a) Color-coded linear CSRS map performed at the organic layer. (b)-(c) Representative normalized Raman spectra of the non-emissive and emissive spots with the corresponding deconvoluted bands, respectively.

\section{ACKNOWLEDGMENTS}

BAPS is indebted to $\mathrm{K}$. Schröter and $\mathrm{C}$. Brandstätter at Nanoident Technologies AG. We would like to thank Dago de Leeuw for providing the samples and to Fundação para a Ciência e Technology, research unit 631, CEOT, and QUBITeXp International Trade S.A.S. Colombia.

\section{REFERENCES}

1. J. S. Kim, P. K. H. Ho, C. E. Murphy, N. Baynes and R. H. Friend, Adv. Mater. 14, 206-209 (2002).
2. E. Giorgetti, G. Margheri, T. Delrosso, S. Sottini, M. Muniz-Miranda, M. Innocenti, Appl. Phys. B 79, 603609 (2004).

3. J. C. Scott, J. H. Kaufman, P. J. Brock, R. DiPietro, J. Salem, and J. A. Goitia, J. Appl. Phys. 79, 2745-2751 (1996).

4. J. Steiger, S. Karg, R. Schmechel, H. von Seggern, Synth. Met. 122, 49-52 (2001),

5. Y. Furukawa, J. Phys. Chem. 100, 15644-15653 (1996).

6. G. Salvan, B. A. Paez, D. R. T. Zahn, L. Gisslen, and R. Scholz, "Metal/Organic Interface Formation Studied In Situ by Resonant Raman Spectroscopy," in Physical and chemical aspects of organic electronics: from fundamentals to functioning devices: structural and electronic properties of OFETs, edited by C. Wöll, Wiley-VCH, Weinheim, 2009, pp. 263-280.

7. M. Cölle, M. Büchel, D. M. de Leeuw, Org. Electr. 7, 305-312 (2006). 